\title{
Effect of Anthocyanins from Purple Corn (Zea mays L.) on DF-1 Cells Ziteng HUANG ${ }^{1,2, a}$, Dan WANG ${ }^{2, a}$, Yue $\mathrm{MA}^{2, \mathrm{~b}}$ Chao Zhang ${ }^{2, c}$, Yubin Wang ${ }^{2, \mathrm{c}}$ and Xiaoyan $\mathrm{ZHAO}^{1,2, \mathrm{c},{ }^{*}}$
}

${ }^{1}$ Food Science and Engineering College, Beijing University of Agriculture, Beijing, 102206, China;

${ }^{2}$ Beijing Vegetable Research Center, Beijing Academy of Agriculture and Forestry Science, Key La boratory of Biology and Genetic Improvement of Horticultural Crops (North China), Ministry of Agri culture, P.R.China, Key Laboratory of Urban Agriculture (North), Ministry of Agriculture, Beijing Ke y Laboratory of agricultural products of fruits and vegetables preservation and processing,P.R. Beijing, 100097, China;

awangdan@nercv.org, ${ }^{b}$ mayue@nercv.org, ${ }^{\mathrm{c}}$ zhaoxiaoyan@nercv.org

\section{Keywords: Purple corn, Anthocyanins, MTT assay}

\begin{abstract}
This study investigates the composition and content of anthocyanins, which are from the purple corn (Zea mays L.) bracts. The content of purple corn anthocyanins (PCA) is $17.13 \%$. Results of the identification of PCA showed that the most prominent of the all components was cyanidin type. DF-1 cells were treated with different concentrations of PCA at different time point. We observed that $50 \mu \mathrm{g} / \mathrm{mL}$ PCA was the optimal concentration, which had no cytotoxicity to DF-1 cells. The situation of cell growth was evaluated by optical microscope, cell counter, and MTT assays.
\end{abstract}

\section{Introduction}

Purple corn is a kind of anthocyanins food material, showing purple color, regardless of its bracts or grain. The color of purple corn is due to its anthocyanins [1]. Anthocyanins have been reported to have many biological functions, such as antioxidant, antimicrobial, antiobesity, and anticancer activities [2]. Chicken embryo fibroblasts have normal fibroblastic morphology and which are free of endogenous sequences related to avian sarcoma and leukosis viruses [3]. DF-1 is a continuous cell line of chicken embryo fibroblasts and it serves as a research model of avian leukosis. Purple corn is a kind of chicken feed. However, the effect of PCA to DF-1 cells has not been investigated yet. This work is to find out the optimal concentration of PCA to DF-1 cells living. Moreover, cells activity was explored by MTT assay.

\section{Materials and Methods}

Reagents and chemicals. Purple corn bracts and DF-1 cells line were supplied by Beijing Academy of Agriculture and Forestry Sciences. Dulbecco's Modified Eagle Medium (DMEM), fetal bovine serum (FBS) and Penicillin-Streptomycin Solution (100x) were supplemented by Gibco, (Shanghai, China); 3-(4,5-Dimethylthiazol-2-yl)-2,5-diphenyltetrazolium bromide (MTT) was purchased from Sigma (Shanghai, China). Trypsin-EDTA solution was produced from Wuhan Fine Biotechnology Company (Wuhan, China), dimethyl sulfoxide (DMSO) was purchased from Solarbio (beijing, China).

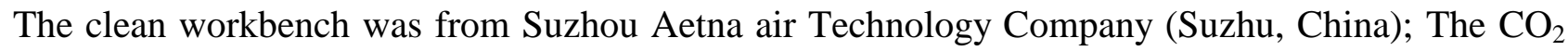
constant temperature incubator was obtained from Thermo Scientific Company (Boston, Massachusetts, USA). The microplate reader SpectraMax i3 was purchased from Molecular Devices Company (USA). The Cell counter was purchased from Wuzhou Eastern Company (Beijing, China). The 3K15 centrifugal machine was from Sigma Company (Shanghai, China).

Preparation of total anthocyanins from the purple corn bracts. The dried purple corn bracts were extracted with $60 \%$ (v/v) ethanol acidified with hydrochloric acid until $\mathrm{pH}=3.0$ at $50{ }^{\circ} \mathrm{C}$ for $120 \mathrm{~min}$. The ratio of purple corn and extracted solution was 1:65 (v/v). The supernatants were evaporated to dryness at $45^{\circ} \mathrm{C}$ with a rotary evaporator. It was filtered through a macroporous resin to remove the 
sugars, organic acids, phenolic acids and flavonoids, respectively. Finally, the filtrate was dried in the freeze-drying machines to get anthocyanins powder, stored at $-80^{\circ} \mathrm{C}$. The concentration of anthocyanins was detected by $\mathrm{pH}$ differential method.

The recovery of DF-1 cell. Preheat the DMEM supplemented with $15 \%$ FBS, sodium penicillin(100 $\mathrm{U} / \mathrm{mL})$ and streptomycin $(100 \mu \mathrm{g} / \mathrm{mL})$. The cell cryopreservation tube was taken out from the liquid nitrogen and it was shaken in the warm water at $37^{\circ} \mathrm{C}$ rapidly, ice dissolved quickly, cells were centrifuged at 1000r for $5 \mathrm{~min}$, the supernatant was discarded, the preheated medium was added and cells were aspirated by gently pipetting. All the cells were seeded into cell culture flasks $\left(25 \mathrm{~mm}^{2}\right)$. The cells were maintained at $37{ }^{\circ} \mathrm{C}$ in the atmosphere of $5 \% \mathrm{CO}_{2}$ and $95 \% \mathrm{O}_{2}$, the cells were allowed to attach for $24 \mathrm{~h}$.

The subculturing of DF-1Cells. The culture medium was removed and discarded, rinsed the cell layer with 5.0 mL PBS to remove all traces of serum. Then, $2.0 \mathrm{~mL}$ Trypsin-EDTA solution was added to flask and cells were observed under an inverted microscope until cell layer was dispersed. After that, $6.0 \mathrm{~mL}$ complete growth medium was added and cells were aspirated by gently pipetting. Finally, the cells were incubated at $37^{\circ} \mathrm{C}$.

Effect of PCA for DF-1 cell growth. The PCA powder was diluted by $0.01 \mathrm{~mol} / \mathrm{L}$ PBS until the concentration was $12.5 \mathrm{mg} / \mathrm{mL}$, and filtered by a $0.22 \mu \mathrm{m}$ syringe filter. The DF-1 cells were cultured by DMEM containing 10\% FBS and antibiotics, they were seeded in 96-well plates at a density of $5.79 \times 10^{5}$ cells/well at $37^{\circ} \mathrm{C}$ with $5 \% \mathrm{CO}_{2}$. The culture medium was removed until forming monolayer, and it was replaced with DMEM containing 2\% FBS. Cells in each well were treated with PCA (50, $75,100,150 \mu \mathrm{g} / \mathrm{mL}$ ) for $12,24,36,48,60,72,96,120$ and $144 \mathrm{~h}$. Cells were recorded with cell counter at the same time. Three replicates for each concentration, set the cell control and repeat controls.

Results criteria as follows: "+++++" represents the best cell growth, cell has good refraction, and form a complete monolayer; "++++" indicates the cells grow well, form a monolayer; "+++" indicates cell growth in general, it cannot form a monolayer, there are much voids and few cell; "++" indicates cell growth poor, it can't present fundamental cellular morphology, a small amount of them adherent on the wall of cell flask; "+" indicates cell growth is poor, there is no basic cell morphology, a small amount of adherent; "-" indicates poor cell growth, all the cells have been broken, incomplete or poorly defined.

Cell viability (MTT assay). To evaluate the cytotoxicity of PCA, cell viability assay was performed by the same procedure as described previously[4]. DF-1 cells grew in DMEM medium containing $10 \%$ FBS, streptomycin $(100 \mu \mathrm{g} / \mathrm{mL})$ and sodium penicillin $(100 \mathrm{U} / \mathrm{mL})$. At the logarithmic growth phase, the cells were trypsinized and resuspended in complete growth medium. Briefly, $1.14 \times$ $10^{5}$ cells suspended in DMEM medium $(200 \mu \mathrm{l})$ containing $10 \%$ FBS were seeded in 96 -well plates and treated with $0,50,75,100,150 \mu \mathrm{g} / \mathrm{mL}$ of PCA. After incubation for 24, 48, 72 and $96 \mathrm{~h}$, MTT $(0.5 \mathrm{mg} / \mathrm{mL})$ was added to each well, the cells were incubated at $37^{\circ} \mathrm{C}$ for an additional 4 hours. Formazan was dissolved in dimethyl sulfoxide (DMSO) and add to each well. The 96 wells plates were agitated for $10 \mathrm{~min}$ at medium speed. Finally, absorbance was measured by multifunctional microplate reader (Molecular Devices company,USA) at $490 \mathrm{~nm}$ [5].

Statistical analysis. All experiments in this paper were repeated at least three times.Data are expressed as the mean $\pm \mathrm{SD}$. The statistical analyses were done with the software origin 8.0.

\section{Results and Discussion}

HPLC measurement of PCA. The identification of PCA composition results were shown in Table 1. It is reported that the PCA belong to cyanidin, peonidin, pelargonidin or delphinidin aglycone, were bound with glucose or rhamonside, with glucose acylated with malonyl group[5]. According to the statistics in Table 1, it is obvious that cyanidin type is the main content of purple corn bracts extract, at about $63.20 \%$, the figure of pelargonidin tpye is about $11.19 \%$, the percentage of peonidin type is about $8.041 \%$. The lowest among all the categories is the delphinidin type, is about $7.931 \%$. 
Table 1 HPLC-DAD results, proportion of compound type in PCA

\begin{tabular}{ccc}
\hline No. & Compound type & Proportion $(\%)$ \\
\hline 1 & Cyanidin type & 63.20 \\
2 & Pelargonidin tpye & 11.19 \\
3 & Peonidin type & 8.041 \\
4 & Delphinidin type & 7.931 \\
\hline
\end{tabular}

Analysis of the effect of PCA on DF-1 cell growth. The PCA of different concentrations affected on DF-1 monolayer growth significantly. The results showed in Table 2. When the cells were cutured before $24 \mathrm{~h}$, the higher the concentration of the anthocyanins was, the better the DF-1 cells grew. After $24 \mathrm{~h}$, the optimal concentration for cell viability was $50 \mu \mathrm{g} / \mathrm{mL}$. Cells were stained with Trypan blue and recorded viability by cell counter (Table 3 ), the results were similar to Table 2 . The results criteria as previously described.

Table 2 Effect of different concentrations of PCA on DF-1 cell growth

\begin{tabular}{cccccc}
\hline \multirow{2}{*}{ Time $(\mathrm{h})$} & \multicolumn{5}{c}{ Concentration $(\mu \mathrm{g} / \mathrm{mL})$} \\
\cline { 2 - 5 } & 50 & 75 & 100 & 150 & \\
\hline 12 & +++++ & +++++ & +++++ & +++++ & +++++ \\
24 & +++++ & +++++ & +++++ & +++++ & +++++ \\
36 & ++++ & ++++ & ++++ & +++ & ++++ \\
48 & ++++ & ++++ & ++++ & +++ & ++++ \\
60 & +++ & +++ & +++ & ++ & +++ \\
72 & +++ & +++ & +++ & ++ & +++ \\
96 & +++ & ++ & ++ & ++ & +++ \\
120 & ++ & + & + & + & ++ \\
144 & + & - & - & - & + \\
\hline
\end{tabular}

Table 3 Effect of different concentrations of PCA on DF-1 cell viability (\%)

\begin{tabular}{|c|c|c|c|c|c|}
\hline \multirow{2}{*}{ Time (h) } & \multicolumn{4}{|c|}{ Concentration $(\mu \mathrm{g} / \mathrm{mL})$} & \multirow{2}{*}{ Control } \\
\hline & 50 & 75 & 100 & 150 & \\
\hline 24 & $95.41 \pm 1.259$ & $95.7 \pm 2.013$ & $96.24 \pm 1.547$ & $96.92 \pm 1.933$ & $98.81 \pm 1.541$ \\
\hline 48 & $93.84 \pm 1.870$ & $94.53 \pm 1.424$ & $89.95 \pm 2.371$ & $94.09 \pm 0.971$ & $96.77 \pm 1.834$ \\
\hline 72 & $91.57 \pm 1.838$ & $83.66 \pm 2.602$ & $84.87 \pm 1.751$ & $80.70 \pm 2.243$ & $89.77 \pm 3.927$ \\
\hline 96 & $86.11 \pm 1.703$ & $79.84 \pm 1.459$ & $80.00 \pm 1.839$ & $76.83 \pm 1.309$ & $83.15 \pm 3.305$ \\
\hline 120 & $96.88 \pm 2.970$ & $93.55 \pm 2.284$ & $93.94 \pm 1.775$ & $92.93 \pm 1.231$ & $84.62 \pm 4.505$ \\
\hline 144 & $86.25 \pm 0.806$ & $85.00 \pm 1.768$ & $78.69 \pm 1.036$ & $85.92 \pm 1.534$ & $68.04 \pm 2.283$ \\
\hline
\end{tabular}

Data are expressed as means $\pm S D, n=3$. Data in parenthesis represent the percentage $(\%)$ of the cell viability.

In order to verify the correctness of the results, we determined to treat DF-1 cells with various concentrations of PCA by MTT assay. The methods as previously described. The results were shown in Fig.1. Compared to the controls, the higher the concentration of PCA was, the better promoting effect to the cell grew, within $24 \mathrm{~h}$. The treatment of $50 \mu \mathrm{g} / \mathrm{mL}$ PCA was similar to the controls, which had no cytotoxicity to DF- 1 cells. When the concentration of PCA over $50 \mu \mathrm{g} / \mathrm{mL}$, it showed a strong inhibitory effect for cells growth. 


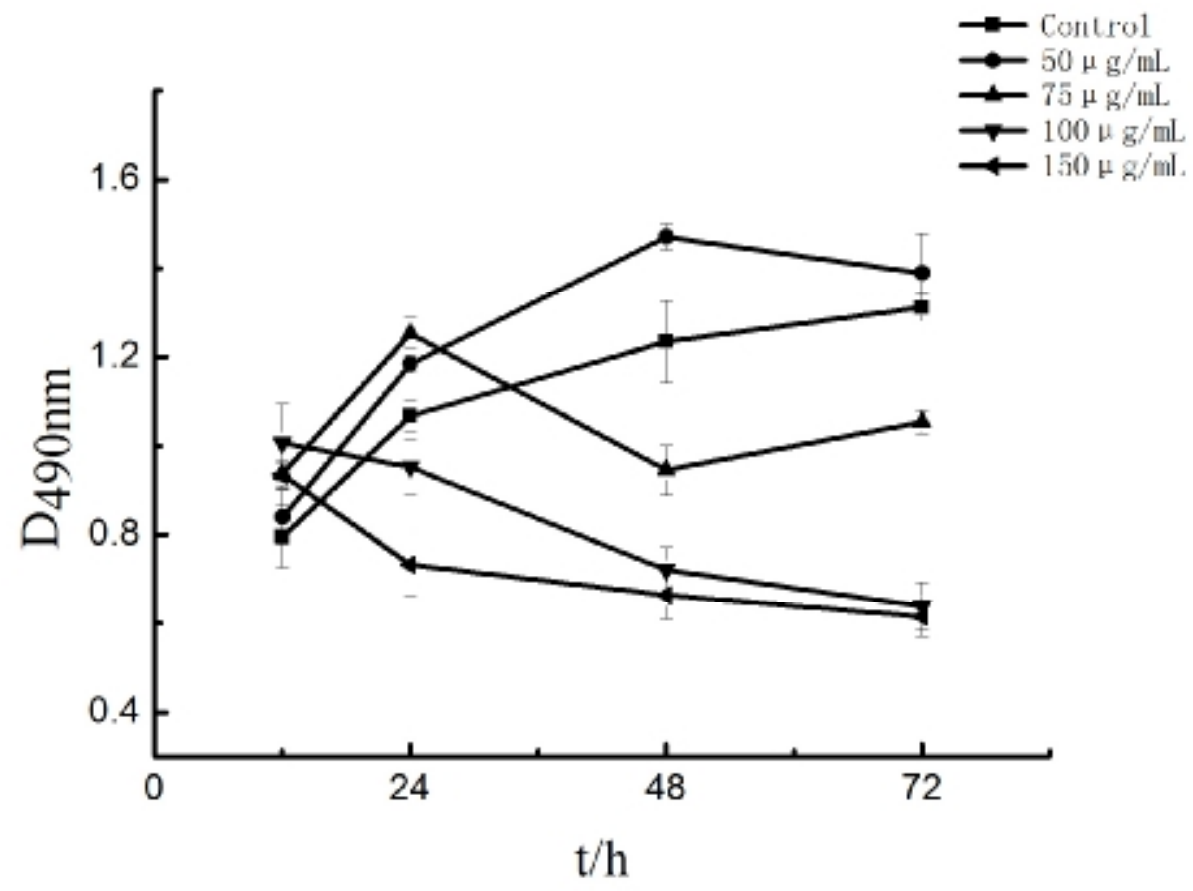

Fig.1-Effect of PCA for DF-1 cell growth by MTT assay

\section{Summary}

In summary, the results suggest that cyanidin type is the major compound in PCA. The optimal concentration of PCA for cell viability is $50 \mu \mathrm{g} / \mathrm{mL}$, when the concentration of PCA below $50 \mu \mathrm{g} / \mathrm{mL}$, it promotes DF-1 cells growth. On the contrary, it inhibits the cell growth. These will provide data support for the research of DF-1 cells in the future.

\section{Acknowledgements}

This work was supported by Beijing Nova program (Z131105000413023) and China Agriculture Research System (CARS-25).

\section{References}

[1] Xiaoyan Zhao, Chao Zhang, Claudia Guigas , Yue Ma, Margarita Corrales, Bernhard Tauscher, Xiaosong $\mathrm{Hu}$, Composition, antimicrobial activity, and antiproliferative capacity of anthocyanin extracts of purple corn (Zea mays L.) from China, European Food Research and Technology. 228(2009) 759-765

[2] Katsube N, Iwashita K, Tsushida T, Yamaki K, Kobori M, J Agric Food Chem 51(2003) 68 - 75

[3] Martin Himly, Douglas N. Foster,Ivan Bottoli, Jason S. Iacovoni. Peter K.Vogt, The DF1 Chicken Fibroblast Cell Line: Transformation Induced by Diverse Oncogenes and Cell Death Resulting from Infection by Avian Leukosis Viruses. Virology. 2(1998) 295-304

[4] Martin, A., Morcillo, N., Lemus, D., et al., 2005. Multicenter study of MTT and resazurin assays for testing susceptibility to firstline anti-tuberculosis drugs. Int. J. Tuberc. Lung Dis. 9, 901-06.

[5] Dan Wang, Yongdong Lei, Yue Ma, Li Zhang, Xiaoyan Zhao, Inhibition of ALV-A-induced apoptosis in DF-1 cells via inactivation of nuclear transcription factor $\mathrm{\kappa} B$ by anthocyanins from purple corn (Zea mays L.)Journal of Functional Foods. 10(2014) 274-282. 